\title{
Theoretical basis of ultimate limit state design
}

\author{
C. C. SMITH*
}

\begin{abstract}
This letter proposes a fundamental theoretical framework for undertaking ultimate limit state design based on the use of an interaction diagram or yield surface. Conceptually (using Eurocode terminology), the design state is obtained from the characteristic state, not by applying partial factors, but by applying a perturbation force or stress probe within this yield surface until factored actions become equal to factored resistances. This robustly ensures mechanical rigour at all times. The method gives clarity as to how calculations should be carried out and removes ambiguity about factoring actions not in the direction of the perturbing force. Several examples are given to illustrate its application.
\end{abstract}

KEYWORDS: limit state design/analysis

ICE Publishing: all rights reserved

\section{INTRODUCTION}

Many modern geotechnical design codes, such as Eurocode 7 (BSI, 2004) are based on limit state design principles and utilise partial factors on actions, resistances and material properties to determine whether a design is safe or unsafe. While at the present time there is broad consensus on how to undertake ultimate limit state (ULS) design and apply partial factors for specific design problems, a general framework appears to be lacking. Smith \& Gilbert (2011a, $2011 \mathrm{~b}$ ) highlighted a number of anomalies and variances in opinion in the literature relating to ULS design calculations and proposed some general practical design principles. Building upon this work, this letter describes a simple but fundamental theoretical framework that should underpin ULS design and permits the definition of a rigorous and consistent methodology for the application of partial factors. This will be illustrated through the application of the framework in two design problems.

The language and terminology used in this note is taken from Eurocode 7, but the principles described should be applicable to any limit state design code that utilises partial factors.

\section{DESIGN STATES AND DESIGN ASSESSMENT}

Common states discussed in limit state design (following Eurocode terminology) are

- characteristic state (KS)

- design state (DS)

- ultimate limit state (ULS).

Conceptually, it is usually taken that the DS is derived from the KS through the application of partial factors and for ULS design it is necessary to show that the DS does not violate yield. Diagrammatically, this can be depicted by the states in Fig. 1. While the KS and DS are points, the ULS is a (multi-dimensional) surface as the system can, in general, fail in many different ways.

Manuscript received 16 July 2012; first decision 30 August 2012; accepted 27 September 2012.

Published online at www.geotechniqueletters.com on 7 November 2012.

*Department of Civil and Structural Engineering, University of Sheffield, Sheffield, UK
For a statically determinate structural problem such as the bending of a simply supported beam under a centrally applied vertical load, use of this system of states is a straightforward and unambiguous process. The applied load $(\mathrm{KS})$ is factored as appropriate to give the DS in which state the maximum bending moment can be determined. The design resistance is the factored plastic moment of resistance of the beam. A check is then made that factored actions are less than or equal to factored resistances. It is noted that the resistance is technically that available at the ULS, and not that actually provided by the beam at the DS (otherwise the assessment would always return an unsafe result). Hence, the DS is inside the ULS yield surface.

In the above example, both the nature and magnitude of the action and resistance are known in advance of determination of the collapse mechanism. (In this note, the term 'actions' will be used to describe both actions and action effects.) However, in a more general case, actions and resistances are specific to a specified stability assessment (e.g. active and passive pressures in a sliding assessment of a gravity retaining wall) and their magnitudes can only be found after the collapse mechanism has been identified. Smith \& Gilbert (2011a) discuss these issues in detail and also demonstrate that mechanical equilibrium is normally lacking in certain common conventional analyses such as the retaining wall example above, rendering the result questionable. What is required is a mechanically rigorous approach.

\section{PROPOSED DESIGN ASSESSMENT}

Stress probes and yield surface

Yield surfaces or interaction diagrams (e.g. Butterfield, 2012) are increasingly being used as a robust approach for assessing geotechnical stability. Such a yield surface may be defined in stress or force space and may be determined through the use of stress or force probes. Hence to determine the yield surface for a footing subjected to combined $\mathrm{V}-\mathrm{H}$ loading, loads of various inclinations are applied to the footing and increased until yield is obtained. These probes may be applied relative to the KS. Probes are typically applied to a defined structural element within a soil body (e.g. a footing or a wall).

If the system exhibits perfect plasticity and obeys an associative flow rule then this yield surface is unique. If not, 


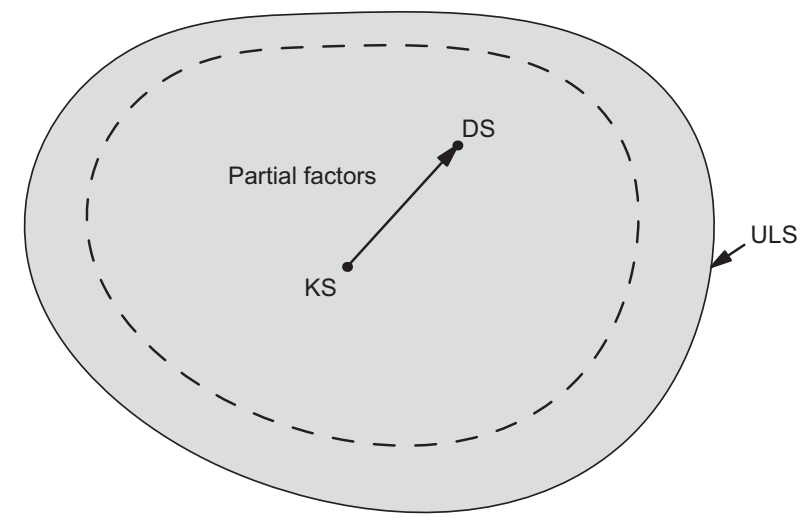

Fig. 1. Design states: characteristic state (KS), design state (DS) and ultimate limit state (ULS)

then variation in the load path to failure could result in a different yield value. For simplicity in this note, stress probes will be defined to be monotonic and linear (i.e. are applied in a monotonically increasing value in the same direction), as shown in Fig. 2.

By comparing Figs 1 and 2, it can be seen that the path from the KS to the DS can be viewed as part of the stress probe path to the ULS. Considered this way, the DS becomes a mechanically rigorous state (i.e. is in equilibrium if the stress probe itself is included). It is therefore not the state where factors are applied to the KS, but it is the state where a suitable stress probe is applied which gives rise to the condition that factored actions (excluding probe) equal factored resistances in the direction of the probe. Crucially, these actions and resistances can include forces (e.g. earth pressure resultants) that can only be determined as a result of stress probe analysis.

It is important to note that, in this framework, actions are forces acting on the structural element resolved parallel to and in the direction of the probe, while resistances are those forces resolved parallel to but opposing the direction of the probe.

It is therefore seen that a DS can only be assessed for a specific loading direction or stress probe direction, and is only valid for that direction. To fully assess the safety of a geotechnical construction it would be necessary to consider probes in all possible directions. However, in conventional practice, only a few key directions are typically considered (e.g. sliding, bearing and overturning of a gravity wall) and this may miss a critical mode of failure.

If factoring of material strength parameters is also to be included within this action/resistance factoring framework, then this simply requires the adoption of a contracted yield

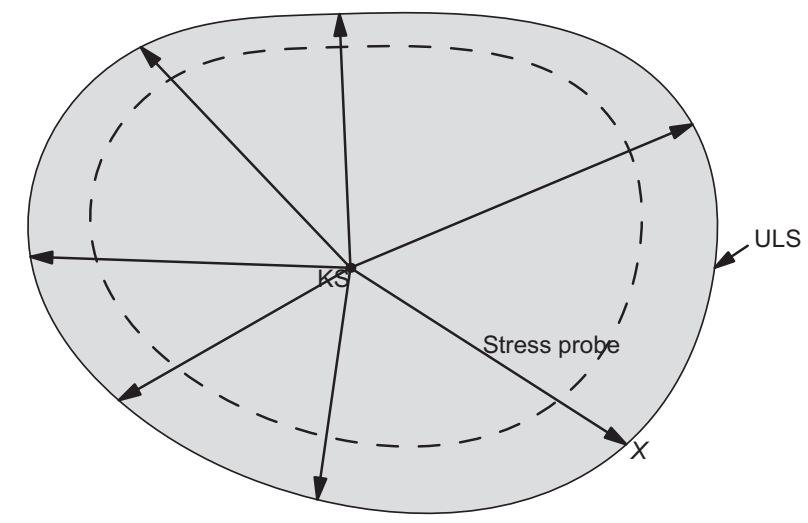

Fig. 2. Definition of ULS surface using stress probes surface (e.g. the dashed surface in Figs 1 and 2) and the design check requires that the DS is shown to lie inside this contracted ULS surface, rather than the original ULS surface.

An alternative view of the above that is perhaps more appropriate to the term 'ULS design' is to continue the probe until ULS is achieved. Then, it is simply necessary to show that the factored actions are less than or equal to the factored ULS resistances.

\section{Special case: material factoring}

In most material factoring approaches (e.g. Eurocode 7 Design Approach 1 Combination 2 (DA1/2) (BSI, 2004)), actions and resistances are not generally factored (i.e. DS = KS). Thus, in the conceptual model presented in Fig. 2, when a stress probe (in any direction) is applied, the unfactored actions will always be less than the unfactored resistances (since equilibrium dictates that actions plus probe equal resistances). Safety is thus ensured if the DS can be shown to lie within this contracted ULS surface. This can be determined numerically by showing that the DS does not lead to collapse. Alternatively, for a perfectly plastic material, the lower bound theorem may be adopted: if a stress field can be found that nowhere violates yield and satisfies the boundary conditions then the system is inside the ULS surface. The significant advantage of this approach is that it automatically considers all possible failure modes in one calculation, since no failure direction is specified.

Furthermore, action factors may be adopted on the condition that they only apply to pre-defined actions of fixed magnitude (e.g. an external dead load). Then, the DS is a fixed offset from the KS and the above approach can still be applied. This is the situation with Eurocode 7 DA1/ 2 and Design Approach 3, where either only unfavourable actions or structural actions are factored, respectively. In general, these are pre-defined and independent of the collapse mode (but not always).

\section{EXAMPLES}

\section{Sliding failure of gravity retaining wall}

When designing a gravity retaining wall such as depicted in Fig. 3 against sliding using action/resistance factoring, it is

(a) not necessarily clear how to determine the ULS values of $\boldsymbol{A}, \boldsymbol{P}, \boldsymbol{T}, \boldsymbol{F}_{A}, \boldsymbol{F}_{P}$ and $\boldsymbol{N}$ using a numerical model and

(b) while it is clear how to factor the action effect $\boldsymbol{A}$ and the resistances $\boldsymbol{P}$ and $\boldsymbol{T}$, it is not clear how $\boldsymbol{F}_{A}, \boldsymbol{F}_{P}$ and $\boldsymbol{N}$ should be factored. For example, $\boldsymbol{F}_{A}$ could be taken as an unfavourable action effect as it is part of the active force $\boldsymbol{A}$ (invoking the single source principle (BSI, 2002) or it could be considered as favourable as it increases $N$ and thus $\boldsymbol{T}$.

Application of the proposed framework requires that

(a) limiting ULS stress states and thus appropriate actions and resistances can be induced by causing the wall to slide by an external stress probe (horizontal force pulling the wall to the left)

(b) none of the other forces $\boldsymbol{F}_{A}, \boldsymbol{F}_{P}$ and $\boldsymbol{N}$ should be factored as they act perpendicular to the stress probe direction.

It is straightforward to show that factoring of $\boldsymbol{F}_{A}, \boldsymbol{F}_{P}$ or $\boldsymbol{N}$ is a theoretically invalid process. Factoring any of these forces would only be relevant to the stability assessment if this subsequently lead to a change in $\boldsymbol{T}$ (or $\boldsymbol{P}$ or $\boldsymbol{A}$ ) via a mechanics calculation (e.g. $\boldsymbol{T}=\boldsymbol{N} \tan \varphi^{\prime}$ ). However, as soon as a non-unity factor is used, Newton's third law is broken and any mechanics calculation becomes invalid. (This principle does not necessarily apply to factoring of external 


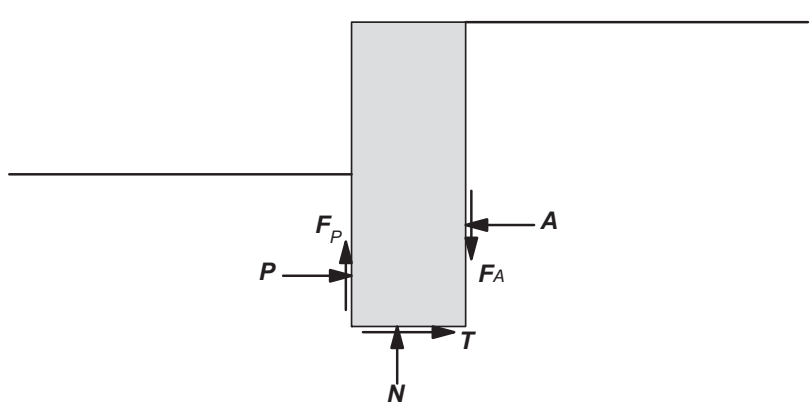

Fig. 3. Gravity retaining wall example

actions known in advance of the calculation. Some issues of debate remain relating to the factoring of such actions that are not parallel to the stress probe direction, such as application of the single source principle and the different factoring of variable and permanent actions. Some discussion of this issue is given in Smith \& Gilbert (2011b).)

\section{Propped sheet pile wall}

Consider the calculations required for determining the safety of a smooth propped sheet pile wall, depicted in Fig. 4, in terms of prop load and moment resistance using action/resistance factoring. For the purposes of illustration and simplicity, the wall will be assumed to remain rigid and the calculation model adopted will assume Rankine active or passive conditions either side of the wall with no arching effects and rigid-plastic behaviour of the wall and prop. The wall is longer than required for rotational stability according to a free earth analysis and thus requires a fixed earth approach. However, this is not kinematically correct for a rigid wall and prop. A free earth analysis cannot be applied for the full length wall as it leads to a nonequilibrium state. The values of the prop load and moment could be conventionally (and conservatively) assessed based on a free earth analysis of a shorter equilibrium wall (Fig. 5(a)) or on a mobilised strength $\left(\varphi^{\prime}=28 \cdot 1^{\circ}\right)$ model of the full length wall (Fig. 5(b)), or make use of an elasto-plastic finite-element or Winkler model. The values so determined might then be factored to obtain the design values.

In the proposed framework, ULS design is achieved through the use of stress probes. These are applied as follows until ULS is reached for the following failure modes (required design parameter in brackets).

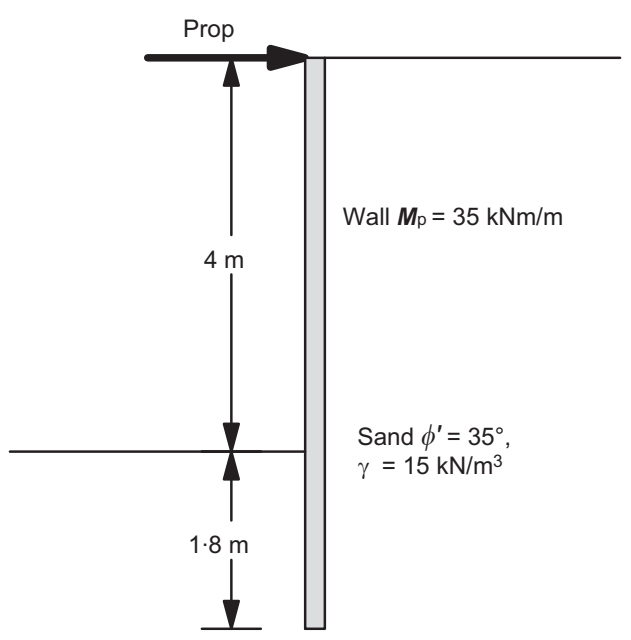

Fig. 4. Propped sheet pile wall embedded in dry sand

$$
1
$$

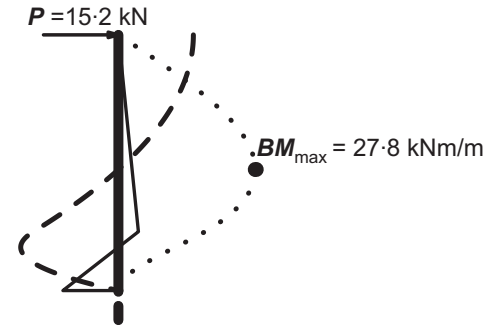

(a)

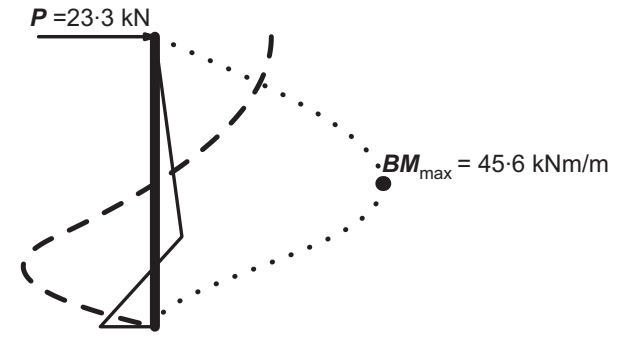

(b)
Fig. 5. Conventional analysis options for determining prop force and maximum bending moment $(\boldsymbol{B} \boldsymbol{M})$ for a propped retaining wall. Failure mechanism is shown on left. Solid line depicts net pressure; dashed line depicts shear force; dotted line depicts bending moment. (a) Conservative 'free earth' design assuming a shorter wall of penetration depth $1.2 \mathrm{~m}$ in equilibrium. Conservative design values may be determined from this calculation. Wall and prop are assumed to remain fully rigid. (b) Mobilised strength approach, using 'free earth' calculation model with reduced $\phi=28 \cdot 1^{\circ}$, to achieve equilibrium for given wall depth $1.8 \mathrm{~m}$. Wall and prop remain fully rigid. In practice, wall flexibility would reduce prop force and moments. (This analysis can also be considered appropriate as a material factoring analysis with a factor on strength of $\tan (35) / \tan (28 \cdot 1)=1 \cdot 31$. This exceeds, for example, the Eurocode DA1/2 factor of 1.25 and therefore would give conservative values of prop force and bending moment for that design approach)

- Case 1: prop stability (prop force). Apply force probe $\boldsymbol{P}_{\text {probe }}$ against prop position (Fig. 6(a)).

- Case 2: failure by wall bending (wall bending moment). Apply local moment increments $\boldsymbol{M}_{\text {probe }}$ at each point down the wall (most critical position shown in Fig. 6(b)).

In each case, the probe generates the required failure mode kinematics (either a prop failure or a bending failure) that enables the other ULS actions and resistances in the problem to be determined which correspond to that failure mode. These in turn can be used to determine the specified design parameter (e.g. the wall bending moment at the ULS $\boldsymbol{M}_{\mathrm{ULS}}$ is given by $\boldsymbol{M}_{\mathrm{ULS}}=\boldsymbol{M}_{\mathrm{p}}-\boldsymbol{M}_{\text {probe }}$ ). The analysis could be carried out using a numerical model. However, for simplicity, a simple fixed earth analysis (i.e. an implicit ULS analysis consistent with the induced kinematics) is used here. This turns out to generate the same stress distributions for each problem due to the highly simplified earth pressure assumptions. (In reality, the active stress distributions in Fig. 6(b) would deviate significantly from Rankine conditions due to, for example, arching effects and would vary with location of the applied probe. These effects would be readily picked up by numerical models.) It can be seen that slightly lower values of moment and prop force are required relative to Fig. 5(a) and significantly lower than Fig. 5(b) due to accounting for the extra length of the wall.

It is important to note that, in both cases, the partial factors are applied only at the point of application of the 

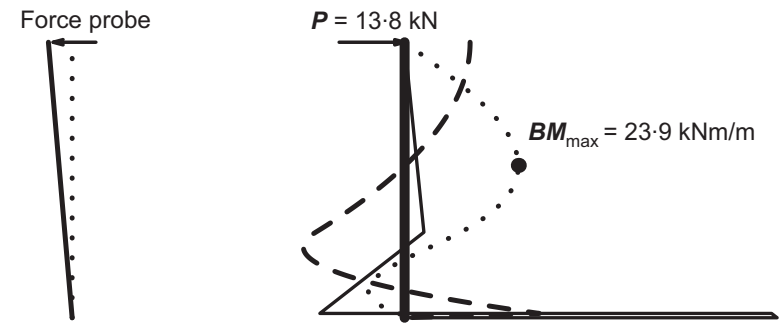

(a)
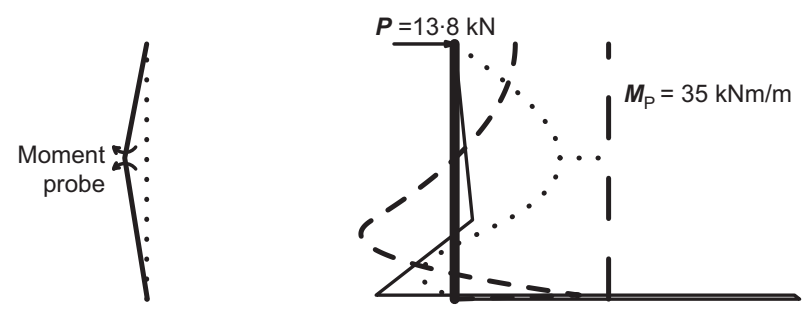

(b)

Fig. 6. Stress probe based approach for determining prop force and maximum bending moment for a propped retaining wall. Solid line depicts net pressure; dashed line depicts shear force; dotted line depicts bending moment. In both cases, the specific collapse kinematics allow an active/passive pressure reversal over a small depth at the base of the wall. (a) ULS method: finding prop force. Stress probe involves application of a force against the prop to induce prop failure and movement of the top of the wall. (b) ULS: bending moment. Stress probe involves application of a matched pair of opposing couples either side of the selected wall position (adding a moment 'spike' at that point) to induce local wall bending

probe. Thus, for example, in Case 2, neither the bending moment nor the plastic moment of resistance (treated as a resistance, not a material property) is factored at any other point on the wall other than at where the probe is applied. Factoring of the action (bending moment) at any other point of the wall would lead to a non-equilibrium state.
However, factoring of the ULS resistances. (assumed a fixed constant value) at any other point would not violate mechanical equilibrium, but would be inconsistent with the general proposed framework and would, it is proposed, be over-conservative.

\section{CONCLUDING REMARKS}

Combination of the interaction diagram or yield surface approach to design with a stipulation that the principles of mechanics should not be violated in an assessment allows a clear unambiguous definition of ULS design procedure. Conceptually, the design state is obtained from the characteristic state, not by applying partial factors, but by applying a perturbation force or stress probe until factored actions equal factored resistances. This ensures mechanical rigour while still applying partial factors. It is then necessary to show that the design state is inside the ULS surface. Alternatively, and perhaps more clearly, the probe can be extended to the yield surface and then it is necessary to show that factored actions are less than or equal to factored ULS resistances. The method gives clarity as to how calculations should be done and removes ambiguity about factoring actions not in the direction of the force probe.

\section{REFERENCES}

BSI (2002) Eurocode - Basis of structural design. London: BSI. BSI (2004) BS EN 1997-1: 2004 Geotechnical design. General rules. London: BSI.

Butterfield, R. (2012). On dimensioning the base of a traditional retaining wall. Géotech. Lett. 2, April-June, 25-28.

Smith, C. C. \& Gilbert, M. (2011a). Ultimate limit state design to Eurocode 7 using numerical methods. Part I: methodology and theory. Ground Engng 44, No. 10, 25-30.

Smith, C. C. \& Gilbert, M. (2011b). Ultimate limit state design to Eurocode 7 using numerical methods. Part II: proposed design procedure and application. Ground Enging 44, No. 11, 24-29.

\section{WHAT DO YOU THINK?}

To discuss this paper, please email up to 500 words to the editor at journals@ice.org.uk. Your contribution will be forwarded to the author(s) for a reply and, if considered appropriate by the editorial panel, will be published as a discussion. 